


\title{
NECESSARY CONDITIONS AND SUFFICIENT CONDITIONS FOR DISFOCALITY AND DISCONJUGACY OF A DIFFERENTIAL EQUATION
}

\author{
URI ELIAS
}

\begin{abstract}
A necessary and sufficient criterion for the $(k, n-k)$ disfocality of the equation $y^{(n)}+p(x) y=0, a<x<b$, is proved. This criterion is used to establish explicit necessary conditions for disfocality in terms of lower order equations and integral inequalities. The same criterion is used to obtain sufficient conditions, expressed by inequalities of similar type.
\end{abstract}

1. Introduction. An $n$th order linear differential equation

$$
y^{(n)}+p_{i}(x) y^{(n-1)}+\cdots+p_{n}(x) y=0,
$$

whose coefficients are continuous on an interval $I$, is said to be disconjugate on $I$ if none of its nontrivial solutions has $n$ zeros in $I$ (including multiplicities). If an equation is not disconjugate on $[a, b]$, the conjugate point of $a$ is defined as the infimum of the values $t, t>a$, such that the equation is not disconjuate on $[a, t]$. If the conjugate point of $a$ exists, it is denoted by $\eta(a)$. There exists a solution associated with the interval $[a, \eta(a)]$, which has a zero of multiplicity $k$ at $x=a$ and a zero of multiplicity at least $n-k$ at $x=\eta(a)$ for certain $k, 1 \leqq k \leqq n-1$, and which does not vanish in $(a, \eta(a))$.

The subject of this paper is the disconjugacy of the equation

$$
y^{(n)}+p(x) y=0,
$$

where $p(x)$ is of constant sign. For (1) we have further information about the solution associated with $[a, \eta(a)]$. It has a zero exactly of multiplicity $k$ at $x=a$ and a zero exactly of multiplicity $n-k$ at $x=\eta(n)$. Moreover, $n-k$ is odd if $p(x) \geqq 0$ and $n-k$ is even if $p(x) \leqq 0[16]$.

The distribution of the zeros of the solution associated with $[a, \eta(a)]$ suggests the following definition: Equation (1) is said to be $(k, n-k)$-disconjugate on an interval $I$ if for every pair of points $a, b \in I, a<b$, there does not exist a nontrivial solution of (1) which satisfies

$$
\begin{array}{ll}
y^{(i)}(a)=0, & i=0, \cdots, k-1, \\
y^{(j)}(b)=0, & j=0, \cdots, n-k-1 .
\end{array}
$$


The least value of $b$ such that there exists a (nontrivial) solution which satisfies (2), is called the $(k, n-k)$-conjugate point of $a$.

Together with $(k, n-k)$-disconjugacy, we consider a related concept: (1) is called $(k, n-k)$-disfocal on $I$ if for every pair of points $a, b \in I, a<b$, there does not exist a nontrivial solution of (1) which satisfies

$$
\begin{array}{ll}
y^{(i)}(a)=0, & i=0, \cdots, k-1, \\
y^{(j)}(b)=0, & j=k, \cdots, n-1 .
\end{array}
$$

The concepts of $(k, n-k)$-disconjugacy and $(k, n-k)$-disfocality are trivial for certain values of $k$. It is well known [16] that there exists a nontrivial solution of (1) which satisfies either (2) or (3) only if $n-k$ is odd and $p(x) \geqq 0$ or $n-k$ is even and $p(x) \leqq 0$. Hence, in the following discussion of $(k, n-k)$-disconjugacy and disfocality we assume that

$$
(-1)^{n-k} p(x)<0 \text {. }
$$

Indeed, for the other values of $k,(1)$ is trivially $(k, n-k)$-disconjugate and disfocal on every interval, disregarding the magnitude of $|p(x)|$.

$(k, n-k)$-disconjugacy and disfocality are connected by the following theorem of Nehari:

THEOREM 1 [18]. If (1) is $(k, n-k)$-disfocal on $(a, b)$, it is $(k, n-k)$-disconjugate on $(a, b)$.

(1) is $(k, n-k)$-disfocal on $(a, \infty)$ if and only if it is $(k, n-k)$ disconjugate on $(a, \infty)$.

For an alternative proof of Theorem 1 , see [1, Lemma 4, Lemma 6].

Theorem 1 is the origin of various necessary criteria and sufficient criteria for disconjugacy. Necessary conditions are obtained according to the scheme

disconjugacy on $(a, \infty) \Rightarrow(k, n-k)$-disfocality on $(a, \infty) \Rightarrow$ coefficient condition ([2], [6], [15], [18], [20]), while sufficient conditions follow from

coefficient condition $\Rightarrow(k, n-k)$-disfocality for every $k \Rightarrow$ $(k, n-k)$-disconjugacy for every $k \Rightarrow$ disconjugacy ([14]).

For example, in [15] the implication $(n-1,1)$-disconjugacy on $(\alpha, \infty) \Rightarrow(n-1,1)$-disfocality on $(a, \infty)$ has been used implicitely, even before $(k, n-k)$-disfocality was defined.

The purpose of this paper is to show that many necessary conditions and sufficient conditions for $(k, n-k)$-disfocality and 
disconjugacy, known conditions as well as new ones, can be derived from one and the same principle.

2. Preliminalies. We shall base our study of $(k, n-k)$ disfocality on the following two characterizations:

TheOREm 2. Equation (1) is (k, $n-k)$-disfocal on $(a, b)$ if and only if it has a solution $y$ which satisfies

$$
\begin{aligned}
y^{(i)}(x)>0, & i=0, \cdots, k-1, \\
(-1)^{j-k} y^{(j)}(x)>0, & j=k, \cdots, n-1
\end{aligned}
$$

on $(a, b)$.

THEOREM 3. Equation (1) is $(k, n-k)$-disfocal on $(a, b)$ if and only if there exist a function $f \in C^{n}(a, b)$ which satisfies

$$
\begin{aligned}
f^{(i)}(x)>0, \quad i=0, \cdots, k-1, \\
(-1)^{j-k} f^{(j)}(x)>0, \quad j=k, \cdots, n-1, \\
(-1)^{n-k}\left[f^{(n)}+p f\right] \geqq 0
\end{aligned}
$$

on $(a, b)$.

Theorem 2 was proved in [1]. For the sake of completeness we shall prove it here again.

First we prove the necessity of (5). (1) has a unique solution (up to a constant multiplicative constant) which satisfies the $n-1$ boundary value conditions

$$
\begin{array}{ll}
y^{(i)}(a)=0, & i=0, \cdots, k-1, \\
y^{(i)}(s)=0, & j=k+1, \cdots, n-1, s>a .
\end{array}
$$

For, if $y_{1}, y_{2}$ are two linearly independent solutions satisfying (7) then there exist a nontrivial solution $y=c_{1} y_{1}+c_{2} y_{2}$ which satisfies (7) and $y^{(k)}(a)=0$. But this is impossible since, as we have remarked above, (1) is $(k+1, n-k-1)$-disfocal if (4) is assumed.

We normalize the essentially unique solution of (7) so that

$$
\sum_{i=0}^{n-1}\left|y^{(i)}(a)\right|=1, \quad y^{(k)}(a)>0,
$$

and in order to emphasize its dependence on $s$, we denote it by $y(x, s)$. An elementary arguments shows that, as result of the uniqueness, $y(x, s), \cdots, y^{(n-1)}(x, s)$ vary continuously with $s$. Actually, $y(x, s)$ can be written explicitely as a $n \times n$ determinant whose elements are the derivatives of $n$ arbitrary, linearly independent solutions of (1). 
When $s$ is sufficiently close to $a, y(x, s), \cdots, y^{(n-1)}(x, s)$ have in $[a, s]$ no zeros except the $n-1$ zeros which are specified in (7). For, if one of them has another zero, then, by repeated application of Rolle's theorem, $y^{(k)}(a, s)$ too has a zero in $[a, s]$. Consequently, we have obtained for an arbitrary small interval a nontrivial solution $y$ such that each of the derivatives $y, \cdots, y^{(n-1)}$ has a zero in the interval, which is impossible. This contradiction confirms that each of $y(x, s), \cdots, y^{(n-1)}(x, s)$ has a fixed sign on $[a, s]$. To determine these signs, observe that by the normalization of $y(x, s)$, we have $y^{(k)}(a, s)>0$ and thus $y^{(k)}(x, s) \geqq 0$ on $[a, s]$; integration and the first part of (7) give the first part of (5). Now, by $(4),(-1)^{n-k} y^{(n)}(x, s)=$ $(-1)^{n-k-1} p(x) y(x, s)>0$; integration and the second part of (7) give the second part of (5). Thus $y(x, s)$ satisfies $(5)$ on $(a, s)$ if $s$ is sufficiently close to $a$.

Let $c$ be the supremum of values of $s, s>a$, such that $y(x, s)$ satisfies (5) on $(a, s)$. If $c=\infty$, the solution $\lim _{s \rightarrow \infty} y(x, s)$ satisfies (5) on $(a, \infty)$ and in this case the necessity part of Theorem 2 follows. If $c<\infty$, it will be proved that (1) is not $(k, n-k)$-disfocal on $[a, c]$, i.e., $c \geqq b$. In this case we shall conclude that the solution $y(x, b)$ satisfies (5).

In order to prove that (1) is not $(k, n-k)$-disfocal on $[a, c]$, we observe that as $s \uparrow c$, we have

$$
\begin{aligned}
y^{(i)}(x, c) \geqq 0, & i=0, \cdots, k-1, \\
(-1)^{j-k} y^{(j)}(x, c) \geqq 0, & j=k, \cdots, n-1, \quad a \leqq x \leqq c .
\end{aligned}
$$

Since $y^{(i)}(x, c), i=0, \cdots, n-1$, are monotonic functions of fixed signs and since $y(x, c) \not \equiv 0$ on every interval, $y(x, c)$ also satisfies (5) on $(a, c)$. On the other hand, as $s \downarrow c$ we obtain by the definition of $c$ that $y(x, c)$ or one of its derivatives has in $[a, c]$ a zero in addition to the $n-1$ zeros which are specified in (7) (for $s=c$ ). Combining $\left(5^{\prime}\right)$ and $(7), y^{(i)}(x, c), i=0, \cdots, k-1$, are found to be increasing positive functions on $(a, c]$ and they cannot have any zero except those given in (7) at $x=a$. Similarly $(-1)^{j-k} y^{(j)}(x, c), j=$ $k+1, \cdots, n-1$, are decreasing, positive functions on $[x, c)$. So the derivative which has in $[a, c]$ a zero not specified in (7) must be $y^{(k)}(x, c)$ and since it is positive on $(a, c)$ and decreasing, its only zero may be only at $x=c$. Therefore (1) is not $(k, n-k)$-disfocal on $[a, c]$. Since $(1)$ is $(k, n-k)$-disfocal on $(a, b)$, it is disfocal on $[a, b)$ too and consequently $b \leqq c$. So $y(x, b)$ satisfies (5) and the necessity part of Theorem 2 is proved.

The necessity part of Theorem 3 follows from the just proved necessity part of Theorem 2, and the sufficiency part of Theorem 2 is a particular case of the corresponding part of Theorem 3. Hence, 
it suffices to establish the sufficient part of Theorem 3 only.

Let $f$ satisfy (6). Suppose that (1) is not $(k, n-k)$-disfocal on $(a, b)$, i.e., there exist two points $a^{\prime}, b^{\prime}, a^{\prime}<b^{\prime}$, in $(a, b)$ and a solution $y$ of (1) which satisfies the boundary conditions (3) at $a^{\prime}, b^{\prime}$. Let $\lambda_{0}$ be the smallest positive value of $\lambda$ such that for some $m, 0 \leqq m \leqq$ $n-1$, the function $(f-\lambda y)^{(m)}$ has a zero in $\left[a^{\prime}, b^{\prime}\right] . \lambda_{0}$ exists since $f>0$ and $y>0$ in $\left(a^{\prime}, b^{\prime}\right)$. Furthermore, $\lambda_{0}>0$ since $f^{(i)} \neq 0$, $i=0, \cdots, n-1$, on $\left[a^{\prime}, b^{\prime}\right]$. In view of inequalities (6) and the definition of $\lambda_{0}$,

$$
\begin{aligned}
\left(f-\lambda_{0} y\right)^{(i)} \geqq 0, & i=0, \cdots, k-1 \\
(-1)^{j-k}\left(f-\lambda_{0} y\right)^{(j)} \geqq 0, & j=k, \cdots, n-1,
\end{aligned}
$$

and

$$
(-1)^{n-k}\left(f-\lambda_{0} y\right)^{(n)} \geqq(-1)^{n-k-1} p\left(f-\lambda_{0} y\right)=|p|\left(f-\lambda_{0} y\right) \geqq 0,
$$

for $a^{\prime} \leqq x \leqq b^{\prime}$ and $\left(f-\lambda_{0} y\right)^{(m)}$ has a zero in $\left[a^{\prime}, b^{\prime}\right]$. Now we shall show that this is impossible.

Indeed, if $0 \leqq m \leqq k-1$, then,

$$
\left(f-\lambda_{0} y\right)^{(m)}\left(a^{\prime}\right)=f^{(m)}\left(a^{\prime}\right)>0
$$

and

$$
\left(f-\lambda_{0} y\right)^{(m+1)}(x) \geqq 0, \quad a^{\prime} \leqq x \leqq b^{\prime},
$$

so $\left(f-\lambda_{0} y\right)^{(m)}$ is an increasing function on $\left[a^{\prime}, b^{\prime}\right]$, positive at $a^{\prime}$, and it does not vanish on $\left[a^{\prime}, b^{\prime}\right]$. If $k \leqq m \leqq n-1$, (3) and (6) give

$$
(-1)^{m-k}\left(f-\lambda_{0} y\right)^{(m)}\left(b^{\prime}\right)=(-1)^{m-k} f\left(b^{\prime}\right)>0 .
$$

Since

$$
(-1)^{m-k}\left(f-\lambda_{0} y\right)^{(m+1)}(x) \leqq 0, \quad a^{\prime} \leqq x \leqq b^{\prime},
$$

$(-1)^{m-k}\left(f-\lambda_{0} y\right)^{(m)}$ is decreasing on $\left[a^{\prime}, b^{\prime}\right]$ and positive at $b^{\prime}$, so has no zeros on $\left[a^{\prime}, b^{\prime}\right]$. This contradiction verifies that $a^{\prime}, b^{\prime}$ with the above properties cannot exist in $(a, b)$ and so $(1)$ is $(k, n-k)$-disfocal on $(a, b)$.

We shall use extensively the following lemma whose first part is due to Kiguradze [7].

LEMMA 1. If

$$
\begin{aligned}
& y^{(i)}(x) \geqq 0, \quad i=0, \cdots, k, \\
& y^{(k+1)}(x) \leqq 0,
\end{aligned}
$$


on $[a, b]$ then

$$
(k-i) y^{(i)}(x) \geqq(x-a) y^{(i+1)}(x), \quad i=0, \cdots, k
$$

on $[a, b]$. If, in addition, $y^{(i)}(a)=0, i=0, \cdots, k-1$, then

$$
(k-i-1) y^{(i)}(x) \leqq(x-a) y^{(i+1)}(x), \quad i=0, \cdots, k-1
$$

and $y^{(i)}(x) /(x-a)^{k-i-1}$ increases on $(a, b)$.

Proof. For $i=k,(10)$ holds trivially since $y^{(k+1)}(x) \leqq 0$. If in the identity

$$
\begin{aligned}
& \int_{a}^{x}\left[(k-i-1) y^{(i+1)}(t)-(t-a) y^{(i+2)}(t)\right] d t \\
& \quad=\left[(k-i) y^{(i)}(x)-(x-a) y^{(i+1)}(x)\right]-(k-i) y^{(i)}(a)
\end{aligned}
$$

we take $i=k-1, k-2, \cdots, 1,0$, (10) follows by an argument of decreasing induction, since, according to $(9), y^{(i)}(a) \geqq 0$. For $k=2$, $i=1$, (10) was proved in [10].

Let now $y^{(i)}(a)=0, i=0, \cdots, k-1$. (11) holds for $i=k-1$ since $y^{(k)}(x) \geqq 0$. Now we have the identity

$$
\begin{aligned}
\int_{a}^{x}[(k & \left.-i-2) y^{(i+1)}(t)-(t-a) y^{(i+2)}(t)\right] d t \\
& =(k-i-1) y^{(i)}(x)-(x-a) y^{(i+1)}(x) .
\end{aligned}
$$

If we take in (12), $i=k-2, k-3, \cdots, 1,0$, (11) follows. Consequently $y^{(i)}(x)(x-a)^{-(k-i-1)}$ increases.

\section{Necessary conditions.}

THEOREM 4. Assume that (1) is (k,n-k)-disfocal on $(a, b)$ and let $1 \leqq q \leqq k, 1 \leqq m-q<n-k$. For every $\alpha, 0 \leqq \alpha \leqq k-q$, the mth order equation $(m<n)$

$$
u^{(m)}+(-1)^{m-q-1} P_{\alpha}(x) u=0,
$$

where

$$
P_{\alpha}(x)=[m ! /(n-1) !](x-a)^{\alpha} \int_{x}^{b}(t-x)^{n-m-1-\alpha}|p(t)| d t,
$$

is $(q, m-q)$-disfocal on $(a, b)$.

Proof. Let $y(x)$ be a solution of (1) which satisfies (5) on $(a, b)$. We consider the $m+1$ consequtive derivatives 


$$
\begin{aligned}
y^{(i)}(x) \geqq 0, & i=k-q, \cdots, k-1, \\
(-1)^{j-k} y^{(j)}(x) \geqq 0, & j=k, \cdots, k+m-q
\end{aligned}
$$

and we shall find a relation between $y^{(k-q)}$ and $y^{(k-q+m)}$.

Taylor's formula with $n-j-1$ terms around the point $b$ for the function $y^{(j)}$ is

$$
\begin{aligned}
y^{(j)}(x)= & \sum_{\mu=0}^{n-j-1}\left(y^{(j)}\right)^{(\mu)}(b)(x-b)^{\mu} / \mu ! \\
& +\int_{b}^{x}(x-t)^{n-j-1}\left(y^{(j)}\right)^{(n-j)}(t) d t /(n-j-1) !
\end{aligned}
$$

We substitute $y^{(n)}=-p y$, exchange the limits of integration and multiply (14) by $(-1)^{j-k}$.

$$
\begin{aligned}
(-1)^{j-k} y^{(j)}(x)= & \sum_{\mu=0}^{n-j-1}(-1)^{j-k+\mu} y^{(j+\mu)}(b)(b-x)^{\mu} / \mu ! \\
& +\int_{x}^{b}(t-x)^{n-j-1}(-1)^{n-k-1} p(t) y(t) d t /(n-j-1) !
\end{aligned}
$$

By (4), $(-1)^{n-k-1} p(t)=|p(t)|$. For $k \leqq j \leqq n-1, a \leqq x \leqq b$, all the terms in (15) are positive according to (5), hence

$$
(-1)^{j-k} y^{(j)}(x) \geqq \int_{x}^{b}(t-x)^{n-j-1}|p(t)| y(t) d t /(n-j-1) !
$$

To obtain lower bounds for $y(t)$ in (16) observe that $y$ increases, therefore for $0 \leqq \nu \leqq 1$,

$$
y(t) \geqq[y(t)]^{\nu}[y(x)]^{1-\nu}, \quad t>x .
$$

By Lemma 1, we have for $0 \leqq i \leqq k$,

$$
\begin{aligned}
y(x) & \geqq(x-a) y^{\prime}(x) / k \geqq \cdots \\
& \geqq(x-a)^{i} y^{(i)}(x) /[k(k-1) \cdots(k-i+1)], \quad x \geqq a .
\end{aligned}
$$

Since $y, \cdots, y^{(k-1)}$ increases, we have for $0 \leqq i \leqq k-1$,

$$
y(t) \geqq(t-x)^{i} y^{(i)}(x) / i !, \quad t \geqq x .
$$

Substituting (18) and (19) into (17), we obtain for $0 \leqq i \leqq k-1$,

$$
\begin{gathered}
y(t) \geqq y^{(i)}(x)(x-a)^{i(1-\nu)}(t-x)^{i \nu}[(k-i) ! / k !]^{1-\nu}[i !]^{\nu}, \\
t \geqq x \geqq a .
\end{gathered}
$$

From (20) and (16) we deduce now that 


$$
\begin{aligned}
(-1)^{j-k} y^{(j)}(x) \geqq & y^{(i)}(x)(x-a)^{i(1-\nu)} \int_{x}^{b}(t-x)^{n-j-1+i \nu}|p(t)| d t \\
& \times\left(\begin{array}{c}
k \\
i
\end{array}\right)^{\nu}(k-i) ! /[k ! \cdot(n-j-1) !] .
\end{aligned}
$$

Finally, we choose $j=k-q+m(\geqq k), i=k-q(\leqq k-1)$ and we set $\alpha=(k-q) \nu$. Since $l ! / r ! \geqq(l+1) ! /(r+1)$ ! whenever $l \leqq r$,

$$
\begin{aligned}
\left(\begin{array}{c}
k \\
i
\end{array}\right)^{\nu} \frac{(k-i) !}{k !(n-j-1) !} & \equiv\left(\begin{array}{l}
k \\
q
\end{array}\right)^{\nu} \frac{q !}{(n-m-1+q-k) ! k !} \\
& \geqq \frac{q !}{(n-m-1+q)} \geqq \frac{m !}{(n-1) !},
\end{aligned}
$$

and (21) gives

$$
\begin{aligned}
& (-1)^{m-q} y^{(k+m-q)}(x) \geqq y^{(k-q)}(x) \\
& \quad \times(x-a)^{\alpha} \int_{x}^{b}(t-x)^{n-m-1-\alpha}|p(t)| d t \cdot m ! /(n-1) !
\end{aligned}
$$

Since $0 \leqq \nu \leqq 1$, we haue $0 \leqq \alpha \leqq k-q$. The function $f(x)=y^{(k-q)}(x)$ satisfies according to (5) and (22),

$$
\begin{array}{r}
f^{(i)}(x)>0, \quad i=0, \cdots, q-1, \\
(-1)^{j-k} f^{(j)}(x)>0, \quad i=q, \cdots, m, \\
(-1)^{m-q}\left[f^{(m)}(x)+(-1)^{m-q-1} P_{\alpha}(x) f\right] \geqq 0
\end{array}
$$

on $(a, b)$. By Theorem 3, (13) is $(q, m-q)$-disfocal on $(a, b)$.

Theorem 4 is valid for $b=\infty$ also. If $(1)$ is $(k, n-k)$-disfocal on $(a, \infty)$ and $y$ is a solution which satisfies $(5)$ on $(a, \infty)$, then $(22)$ holds for every $b, a<b<\infty$. Hence (22) holds for $b=\infty$ also and (13) is $(q, m-q)$-disfocal on $(a, \infty)$. For $m=2, q=1$ compare with Theorem 2 of [12] and Theorem 1 of [13].

THeOREm 5. Let (1) be disconjugate on $(a, \infty)$ and let $2 \leqq m \leqq$ $n-2$. Then the two equations

$$
u^{(m)}+P_{\alpha}(x) u=0, \quad u^{(m)}-P_{\alpha}(x) u=0
$$

(where $P_{\alpha}(x)$ is defined in the statement of Theorem 4), are disconjugate on $(a, \infty)$ for $0 \leqq \alpha \leqq n-m-2$. Furthermore, one of the above two equations, namely

$$
u^{(m)}+[-\operatorname{sgn} p] P_{\alpha}(x) u=0
$$

is disconjugate on $(\alpha, \infty)$ for $2 \leqq m \leqq n-1,0 \leqq \alpha \leqq n-m-1$.

Proof. If (1) is disconjugate on $(a, \infty)$, it is $(k, n-k)$-discon- 
jugate and hence, by Theorem 1 , $(k, n-k)$-disfocal on $(a, \infty)$ for every $k, 1 \leqq k \leqq n-1$. We shall use this property to show that equations (23) are $(q, m-q)$-disfocal and $(q, m-q)$-disconjugate on $(a, \infty)$ for every $q, 1 \leqq q \leqq m-1$. This, in turn, will imply that the above equations are disconjugate.

Let $q, 1 \leqq q \leqq m-1$, be given. One of the equations (23) is trivially $(q, m-q)$-disfocal since the corresponding necessary condition, similar to (4), it not satisfied. For the other equation, we have to find an integer $k$, such that $q \leqq k, m-q<n-k$ and $(-1)^{n-k} p(x) \leqq 0$. We choose $n-k=m-q+1$ or $n-k=m-q+2$ so that (4) will be satisfied. Accordingly $k-q$ will be $n-m-1$ or $n-m-2$ and so we may take $0 \leqq \alpha \leqq n-m-2$ in Theorem 4 . It follows now by Theorem 4 that the appropriate equation of (23) is $(q, m-q)$-disfocal and hence $(q, m-q)$-disconjugate on $(a, \infty)$. Thus equations (23) are disconjugate there.

For (24) we are interested in those values of $q$, which satisfy $(-1)^{m-q}[-\operatorname{sgn} p] P(x) \leqq 0$, i.e., $(-1)^{m-q} p \geqq 0$. For such $q$ we choose $n-k=(m-q)+1$, i.e., $k=q+(n-m-1)$. This choice fits Theorem 4 whenever $n \geqq m+1$ and we may take $0 \leqq \alpha \leqq n-m-1$. The proof now follows by Theorem 4. For $n=3, m=2$, compare with [2].

The proof of the next theorem is analogous to that of Theorems 4 and 5 .

THEOREM 6. If (1) is disconjugate on $(a, \infty)$, then the mth order equation $(2 \leqq m<n)$,

$$
u^{(m)}+[m ! / n !](x-a)^{n-m} p(x) u=0
$$

is disconjugate on $(a, \infty)$.

Proof. It suffices to prove that (25) is $(q, m-q)$-disfocal on $(a, \infty)$ for every $q, 1 \leqq q \leqq m-1$, such that $(-1)^{m-q} p(x) \leqq 0$. Let $q$ be such an integer. Since (1) is disconjugate on $(a, \infty)$, it is $(n-m+q, m-q)$-disfocal on $(a, \infty)$ and it has a solution $y$ such that

$$
\begin{aligned}
y^{(i)}>0, & i=0, \cdots, n-m+q-1, \\
(-1)^{j-(n-m+q)} y^{(j)}>0, & j=n-m+q, \cdots, n .
\end{aligned}
$$

Consider the derivatives $y^{(n-m)}, \cdots, y^{(n)}$. We have

$$
\begin{aligned}
(-1)^{m-q} y^{(n)}(x) / y^{(n-m)}(x) & =(-1)^{m-q-1} \dot{p}(x) y(x) / y^{(n-m)}(x) \\
& =|p(x)| y(x) / y^{(n-m)}(x) .
\end{aligned}
$$


By Lemma 1,

$$
\begin{aligned}
y(x) & \geqq(x-a) y^{\prime}(x) /(n-m+q) \\
& \geqq \cdots \geqq(x-a)^{n-m} y^{(n-m)}(x) \cdot q ! /(n-m+q) !
\end{aligned}
$$

Since $m>q$, we have $q ! /(n-m+q) ! \geqq m ! / n !$ and so by (27),

$$
(-1)^{m-q} y^{(n)}(x) / y^{(n-m)}(x) \geqq|p(x)| \cdot(x-a)^{n-m} m ! / n !
$$

If we denote $f=y^{(n-m)},(26)$ and (28) yield

$$
\begin{gathered}
f^{(i)}>0, \quad i=0, \cdots, q-1, \\
(-1)^{j-q} f^{(j)}>0, \quad j=q, \cdots, m, \\
(-1)^{m-q}\left[f^{(m)}+(m ! / n !)(x-a)^{n-m} p(x) f\right] \geqq 0, \quad a \leqq x \leqq \infty .
\end{gathered}
$$

By Theorem 3, (25) is $(q, m-q)$-disfocal on $(a, \infty)$ for every $q$, $1 \leqq q \leqq m-1$, such that $(-1)^{m-q} p(x) \leqq 0$, hence (25) is disconjugate on $(a, \infty)$. For $n=3, m=2$, see [10]. For $p \geqq 0, m=2$, compare with [4].

The techniques of the proof of Theorem 4 provide more necessary conditions for $(k, n-k)$-disfocality. If in (21) we take $j=k$, $i=k-1$, then

$$
\begin{gathered}
y^{(k)}(x) \geqq y^{(k-1)}(x) \cdot(x-a)^{(k-1)(1-\nu)} \int_{x}^{b}(t-x)^{n-k-1+(k-1) \nu}|p(t)| d t \\
\times k^{\nu} /[k !(n-k-1) !] .
\end{gathered}
$$

By (10), $y^{(k-1)}(x) \geqq(x-a) y^{(k)}(x)$. Substituting this inequatity into (29) and setting $\alpha=1+(k-1)(1-\nu)$, we obtain the necessary condition

$$
\begin{aligned}
& (x-a)^{\alpha} \int_{x}^{b}(t-x)^{n-1-\alpha}|p(t)| d t \\
& \quad \leqq k^{(\alpha-1) /(k-1)} \cdot(k-1) !(n-k-1) !,
\end{aligned}
$$

$a \leqq x \leqq b$, for every $\alpha, 1 \leqq \alpha \leqq k$.

It is well known that (1) is $(k, n-k)$-disfocal if and only if the adjoint equation

$$
y^{(n)}+(-1)^{n} p(x) y=0
$$

is $(n-k, k)$-disfocal. Hence in (30) we may replace $k$ by $n-k$ and obtain the necessary condition

$$
\begin{aligned}
& (x-a)^{\alpha} \int_{x}^{b}(t-x)^{n-1-\alpha}|p(x)| d t \\
& \quad \leqq(n-k)^{(\alpha-1) /(n-k-1)}(k-1) !(n-k-1) !
\end{aligned}
$$


for $\alpha, 1 \leqq \alpha \leqq n-k$. For $n=4, p \leqq 0$, compare (30) and (32) with Theorem 3.3 of [6].

A useful particular case of (30) (for $\alpha=1$ ) is the necessary condition

$$
(x-a) \int_{x}^{b}(t-x)^{n-2}|p(t)| d t \leqq(k-1) ! \quad(n-k-1) !
$$

THEOREM 7. If (1) is $(k, n-k)$-disfocal on $(0, \infty)$ then

$$
x \int_{x}^{\infty} t^{n-2}|p(t)| d t \leqq e^{\cdot}(n-1)(k-1) !(n-k-1) ! \quad 0<x<\infty .
$$

Proof. In (33) we choose $a=0, b=\infty$ and diminish the domain of integration from $(x, \infty)$ to $(\beta x, \infty)$, where $\beta>1$. For $t \in[\beta x, \infty)$ we have $t-x \geqq(1-1 / \beta) t$ and so

$$
\begin{aligned}
(n-k-1) !(k-1) ! & \geqq x \int_{\beta x}^{\infty}(t-x)^{n-2}|p| d t \\
& \geqq(1-1 / \beta)^{n-2} \beta^{-1}(\beta x) \int_{\beta x}^{\infty} t^{n-2}|p| d t
\end{aligned}
$$

for every $0<x<\infty$. Denote $u=\beta x$. Then

$$
u \int_{u}^{\infty} t^{n-2}|p(t)| d t \leqq \beta^{n-1}(\beta-1)^{-(n-2)}(n-k-1) !(k-1) !
$$

for every $0<u<\infty$, and (34) follows if we choose $\beta=n-1$. For comparison note that Nehari proved the necessary condition

$$
x^{\alpha} \int_{x}^{\infty} t^{n-\alpha-1}|p(t)| d t \leqq(n-1)^{2}(k-1) !(n-k-1) ! / \alpha
$$

for $0<\alpha \leqq n-1$ [17, Theorem 5.3]. For even $n$ and $k=n-k$, see [19].

If (1) is disconjugate we take an integer $k$ which satisfies (4) and which minimizes the right hand side of (34). For odd $n$ we choose either $k=(n-1) / 2$ or $k=(n+1) / 2$ and for even $n$, either $k=n / 2$ or $k=n / 2+1$. In either cases, $(k-1) !(n-k-1) ! \leqq$ $[n / 2] ![n / 2-1]$ ! Thus if $(1)$ is disconjugate on $(0, \infty)$ then

$$
x \int_{x}^{\infty} t^{n-2}|p(t)| d t \leqq e(n-1)[n / 2] ![n / 2-1] ! \quad 0<x<\infty
$$

(36) is stronger than the condition (7.10) or (7.12) of [18] and of course, than that of [15].

The constants on the right hand side of (30) and (32) increase with $\alpha$, while the right hand side of (35) is a decreasing function 
of $\alpha$. This phenomenon is not unnatural. Indeed, for $a=0, b=\infty$, and $p(x)=c x^{-n}$, the integrals in (35) and (30) are

$$
\begin{aligned}
& x^{\alpha} \int_{x}^{\infty} t^{n-\alpha-1} \cdot c t^{-n} d t=c / \alpha, \\
& \quad x^{\alpha} \int_{x}^{\infty}(t-x)^{n-\alpha-1} \cdot c t^{-n} d t=c \Gamma(\alpha) \Gamma(n-\alpha) / \Gamma(n) .
\end{aligned}
$$

$\Gamma(\alpha) \Gamma(n-\alpha)$ increases for $\alpha \leqq n / 2$, and since $\min \{k, n-k\} \leqq n / 2$, the left hand side of at least one of (30) and (32) increases.

We do not know what is the best (smallest) constant $N(\alpha)$ which can be taken on the right hand side of (30) (or (35)) to provide a necessary condition for $(k, n-k)$-disfocality. However, for $\alpha=$ $(\beta+\gamma) / 2$ we have

$$
\begin{aligned}
{\left[(x-a)^{\alpha} \int_{x}^{b}(t-x)^{n-\alpha-1}|p| d t\right]^{2} \leqq } & (x-a)^{\beta} \int_{x}^{b}(t-x)^{n-\beta-1}|p| d t \\
& \times(x-a)^{\gamma} \int_{x}^{b}(t-x)^{n-\gamma-1}|p| d t .
\end{aligned}
$$

Hence $[N(\beta+\gamma) / 2)]^{2} \leqq N(\beta) \cdot N(\gamma)$. Both the constants in (30) and (35) satisfy this inequality.

4. Sufficient conditions. Theorems 2 and 3 enable us to obtain sufficient conditions for $(k, n-k)$-disfocality of (1). Our next theorem generalizes Theorem 5.1 of [18] and suggests also a simple proof to that theorem.

THEOREM 8. Given $q, k \leqq q \leqq n-1$. (1) is (k, $n-k)$-disfocal on $(a, b)$ if and only if there exists a nonnegative function $R \in C^{q}$ which satisfies $R^{(i)}(a) \geqq 0, i=0, \cdots, k-1,(-1)^{j-k} R^{(j)}(b) \geqq 0, j \equiv$ $k, \cdots, q-1$ and

$$
(-1)^{q-k} R^{(q)}(x) \geqq \int_{x}^{b}(t-x)^{n-q-1}|p(t)| R(t) d t /(n-q-1) !
$$

Proof. For $q=k$, this is Theorem 5.1 of [18]. To prove the necessity part of the theorem we take $R(x)$ as a solution $y$ of (1) which satisfies (5). (38) follows then from (16) for $j=q$.

To prove the sufficiency part of the theorem, we integrate (38) repeatedly on $[x, b]$. By the assertion $(-1)^{j-k} R^{(j)}(b) \geqq 0, \quad j=$ $k, \cdots, q-1$, we obtain after $q-k$ integrations

$$
R^{(k)}(x) \geqq \int_{x}^{b}(t-x)^{n-k-1}|p(t)| R(t) d t /(n-k-1) !
$$


(which, actually, is the assumption of Theorem 5.1 of [18]). Integrating $k$ times on $[a, x]$, we obtain

$$
\begin{aligned}
R(x) & \geqq \sum_{i=0}^{k-1} R^{(i)}(a)(x-a)^{i} / i ! \\
& +\int_{a}^{x}(x-s)^{k-1}\left(\int_{s}^{b}(t-s)^{n-k-1}|p(t)| R(t) d t\right) d s /[(k-1) !(n-k-1) !] .
\end{aligned}
$$

Let

$$
f(x)=\int_{\alpha}^{x}(x-s)^{k-1} \int_{s}^{b}(t-s)^{n-k-1}|p(t)| R(t) d t d s /[(k-1) !(n-k-1) !] .
$$

Differentiation gives

$$
\begin{aligned}
& f^{(i)}(x) \geqq 0, j=0, \cdots, k-1, \\
&(-1)^{j-k} f^{(j)}(x) \geqq 0, \quad j=k, \cdots, n-1, \quad a \leqq x \leqq b .
\end{aligned}
$$

By (39), $R(x) \geqq f(x)$, so to the end

$$
(-1)^{n-k} f^{(n)}(x)=|p(x)| R(x) \geqq|p(x)| f(x)=(-1)^{n-k-1} p(x) f(x),
$$

i.e.,

$$
(-1)^{n-k}\left[f^{(n)}+p f\right] \geqq 0,
$$

and $(1)$ is $(k, n-k)$-disfocal by Theorem 3 .

Explicit sufficient conditions can be derived from Theorem 8 by choosing a suitable function $R$. If we set $R(x)=(x-a)^{k-\nu}, 0<\nu<1$, we obtain that if for some integer $q, k \leqq q \leqq n-1$, and $0<\nu<1$.

$$
\begin{aligned}
& (x-a)^{q-k+\nu} \int_{x}^{b}(t-a)^{n-(q-k+\nu)-1}|p(t)| d t \\
& \leqq(n-q-1) ! \prod_{i=1}^{q}|q-k+\nu-i|
\end{aligned}
$$

$a \leqq x \leqq b$, then (1) is $(k, n-k)$-disfocal on $(a, b), a<b \leqq \infty$. Note that for $q=k, \nu=0$ is permitted also. For $q=k$, see (5.20) of [17].

If we set $R(x)=(x-a)^{k-1} \log (x-a)$, we obtain (by using the identity $\left.\left(x^{\mid k-1} \log x\right)^{(k)}=(k-1) ! x^{-1}\right)$ the sufficient condition

$$
\begin{gathered}
(x-a)^{q-k+1} \int_{x}^{b}(t-a)^{n-q+k-2} \log (t-a)|p(t)| d t \\
\leqq(k-1) !(q-k) !(n-q-1) !
\end{gathered}
$$

which generalizes (40) for $\nu=1$.

Our next goal is to obtain sufficient conditions for $(k, n-k)$ disfocality of the form 


$$
(x-a)^{\alpha} \int_{x}^{b}(t-a)^{n-\alpha-1}|p(t)| d t \leqq c(\alpha)
$$

for additional values of $\alpha$. For this purpose we generalize Theorem 4.5 of [3]:

THEOREM 9. Let the equation

$$
y^{(n)}+p_{1}(x) y=0
$$

be $(k, n-k)$-disfocal on $(a, b)$ and let $f(x)$ be a positive, differentiable function such that $f(x) /(x-a)^{k-1}$ is nonincreasing on $(a, b)$. If $\operatorname{sgn}\left[p_{1}(x)\right]=\operatorname{sgn}[p(x)]$ and

$$
\int_{x}^{b} f(t)\left|p_{1}(t)\right| d t \geqq \int_{x}^{b} f(t)|p(t)| d t, \quad a \leqq x \leqq b,
$$

then (1) is $(k, n-k)$-disfocal on $(a, b)$.

If $b=\infty, f(x)$ is nonincreasing and (43) holds, then the disconjugacy of (42) implies the disconjugacy of $(1)$ on $(a, \infty)$.

Proof. The first part of the theorem, with $f \equiv 1$, is proved in Theorem 4.5 of [3] as a consequence of a comparison theorem for an associated eigenvalue problem. That proof is based on the theory of $u_{0}$-positive operators. We shall prove now the theorem as a corollary of Theorem 8.

Let $y(x)$ be a solution of (42) which satisfies (5). By the proof of Theorem 2, we may assume without loss of generality that $y^{(i)}(a)=0, i=0, \cdots, k-1$. According to Lemma $1, y(x) /(x-a)^{k-1}$ is a nondecreasing function hence also

$$
y(x) / f(x)=\left[y(x) /(x-a)^{k-1}\right] \cdot\left[(x-a)^{k-1} / f(x)\right]
$$

is nondecreasing. By (16) we have for $j=n-1$

$$
(-1)^{n-1-k} y^{(n-1)}(x) \geqq \int_{x}^{b}\left|p_{1}(t)\right| y(t) d t
$$

Now we show that

$$
\int_{x}^{b}\left|p_{1}(t)\right| y(t) d t \geqq \int_{x}^{b}|p(t)| y(t) d t .
$$

Indeed, $y / f$ does not decrease, hence we have by (43) and the second mean value theorem,

$$
\begin{aligned}
& \int_{x}^{b}\left|p_{1}(t)\right| y(t) d t-\int_{x}^{b}|p(t)| y(t) d t=\int_{x}^{b}\left[\left|p_{1}\right| f-|p| f\right] \cdot[y / f] d t \\
& \quad=[y(x) / f(x)]_{x}^{\xi}\left[\left|p_{1}\right|-|p| f d t+[y(b) / f(b)] \int_{\xi}^{b}\left[\left|p_{1}\right|-|p|\right] f d t\right. \\
& \quad \geqq[y(x) / f(x)] \int_{x}^{b}\left[\left|p_{a}\right|-|p|\right] f d t \geqq 0 .
\end{aligned}
$$


Therefore

$$
(-1)^{n-1-k} y^{(n-1)}(x) \geqq \int_{x}^{b}|p(t)| y(t) d t .
$$

Hence $R \equiv y$ satisfies the assumptions of Theorem 8 for $q=n-1$ and consequently (1) is ( $k, n-k)$-disfocal.

Our theorem holds even if $b=\infty$, since as $b \rightarrow \infty$, the appropriate limits exist. First, $y(x) / f(x)$ is nondecreasing, hence $y(x) / f(x) \geqq A$ for a certain constant $A>0$. Therefore,

$$
(-1)^{n-1-k} y^{(n-1)}(x) \geqq \int_{x}^{b}\left|p_{1}(t)\right| y(t) d t \geqq A \int_{x}^{b}\left|p_{1}(t)\right| f(t) d t,
$$

and the integrals $\int^{\infty}\left|p_{1}\right| y d t, \int^{\infty}\left|p_{1}\right| f d t$ exist. $\xi$ depends on $b$ and we may choose a sequence $\left\{b_{i}\right\}, b_{i} \rightarrow \infty$, such that $\xi\left(b_{i}\right)$ converges to a limit $(\leqq \infty)$. So, as $b_{i} \rightarrow \infty$, also the terms which contain $\xi$ must converge to finite limits, and the theorem follows.

The last detail of the theorem follows from the fact that (1) is disconjugate on $(a, \infty)$ if and only if it is $(k, n-k)$-disfocal on $(a, \infty)$ for every $k, 1 \leqq k \leqq n-1$.

Let us use Theorem 9 to compare (1) with the equation

$$
y^{(n)}+c(x-a)^{-n} y=0, \quad a<x<\infty .
$$

$y=(x-a)^{r}$ is a solution of (44) if $r$ is a root of the algebraic equation

$$
r(r-1) \cdots(r-n+1)+c=0 .
$$

If (44) has a solution $y=(x-a)^{r}$ with $k-1<r<k$, it is $(k, n-k)$ disfocal on $(a, \infty)$ by Theorem 2 . If we define

$$
A_{k} \stackrel{\text { def }}{=} \max _{k-1<r<k}\{r(r-1) \cdots(r-k+1)|r-k| \cdots|r-n+1|\},
$$

then $(44)$ is $(k, n-k)$-disforcal for $c, 0 \leqq(-1)^{n-k} c \leqq A_{k}$.

We put in (43) $\left|p_{1}(x)\right|=A_{k}(x-a)^{-n}, f(x)=(x-a)^{n-\alpha-1}, n-k \leqq$ $\alpha<\infty$. By Theorem 9 we obtain that if

$$
(x-a)^{\alpha} \int_{x}^{b}(t-a)^{n-\alpha-1}|p(t)| d t \leqq A_{k} / \alpha, \quad a<x<\infty,
$$

then $(1)$ is $(k, n-k)$-disfocal on $(a, b)$.

We have already mentioned that $(1)$ is $(k, n-k)$-disfocal if and only if its adjoint $(31)$ is $(n-k, k)$-disfocal. Since $A_{n-k}=A_{k}$, we may take in (46) values of $\alpha, \min \{k, n-k\} \leqq \alpha<\infty$. The criteria (46) for different values of $\alpha$ cannot be compared with each other, 
since both sides of (46) decrease with $\alpha$. For $n=4, p<0$, compare (46) with Theorem 4.5 of [6].

To summarize, we restate (40) and (46):

COROLLARY. Each one of the inequalities

$$
\begin{aligned}
& (x-a)^{\alpha} \int_{x}^{b}(t-a)^{n-\alpha-1}|p(t)| d t \\
& \leqq \begin{cases}(n-[\alpha+k]-1) ! & \prod_{i=1}^{[\alpha+k]}|\alpha-i| 0 \leqq \alpha<n-k \\
& \alpha \neq 1,2, \cdots, n-k-1 \\
A_{k} / \alpha & \min \{k, n-k\} \leqq \alpha<\infty\end{cases}
\end{aligned}
$$

implies the $(k, n-k)$-disfocality of $(1)$ on $(a, b)$.

Indeed, for $0<\alpha<n-k, \alpha$ noninteger, set in (40) $\alpha=q-$ $k+\nu$ where $0<\nu<1, q=[\alpha+k]$. Then $k \leqq q \leqq n-1$. For $\alpha=0$, (47) follows from (40) for $q=k, \nu=0$ (which is permitted). For $0 \leqq \alpha<1$, compare (47) with (5.20) of [17]. For $n=4, p<0$, see also Theorem 4.5 of [6].

We end this discussion by the following simple bound for $A_{k}$. If $1<k<n-1$, we take in (45) $r=k-1 / 2$ and obtain that

$$
\begin{aligned}
A_{k} & \geqq\{(k-1 / 2) \cdot(k-3 / 2)(5 / 2)(3 / 2)(1 / 2)\}\{(1 / 2)(3 / 2) \cdots(n-k-1 / 2)\} \\
& \geqq(k-1) ! \cdot(3 / 2)^{2}(1 / 2)^{2}(n-k-1) !=(9 / 16) \cdot(k-1) !(n-k-1) !
\end{aligned}
$$

If (47) holds for every $k, 1 \leqq k \leqq n-1,(1)$ is disconjugate. If we choose for example $\alpha=n-1$ and replace $A_{k}$ in (47) by $A=$ $\min \left\{A_{1}, \cdots, A_{n-1}\right\}$ we obtain that

$$
(x-a)^{n-1} \int_{x}^{b}|p(t)| d t \leqq A /(n-1)
$$

implies the disconjugacy of $(1)$ on $(a, b)$. From (48) we have the trivial bound $A \geqq([n / 2-1] !)^{2} / 2$. See also [8], [9].

More necessary conditions and sufficient conditions can be obtained by change of variables in (1).

Lemma 2 [5]. Let $y(x)$ be a solution of (1) and let $\xi=$ $(\alpha x+\beta) /(\gamma x+\delta)$. The function $u(\xi)=(\alpha-\gamma \xi)^{n-1} y((\delta \xi-\beta) /(-\gamma \xi+\alpha))$ is a solution of the equation

$$
\frac{d^{n} u}{d \xi^{n}}+\frac{(\alpha \delta-\beta \gamma)^{n}}{(\alpha-\gamma \xi)^{2 n}} p\left(\frac{\delta \xi-\beta}{-\gamma \xi+\beta}\right) u(\xi)=0 .
$$

If $y(x)$ has a zero of certain multiplicity, then obviously $u(\xi)$ has a zero of the same multiplicity at the corresponding point. Hence (1) is disconjugate on an interval if and only if (45) is dis- 
conjugate on an corresponding interval. Moreover, (1) is $(k, n-k)$ disconjugate if and only if (49) is $(k, n-k)$-disconjugate, provided that $\alpha \delta-\beta \gamma>0$. When $\alpha \gamma-\beta \gamma<0$, i.e., the Mobius transformation inverts the orientation, (1) is $(k, n-k)$-disconjugate if and only if $(49)$ is $(n-k, k)$-disconjugate. The invariance of disfocality is not self evident. However, on an infinite interval $(k, n-k)$-disfocality and $(k, n-k)$-disconjugacy are equivalent. Hence if $\xi=\xi(x)$ maps $\left(a_{1}, \infty\right)$ onto $\left(a_{2}, \infty\right)$, not only disconjugacy survives but disfocality also.

For example, the transformation $\xi-a=(x-a)^{-1}$ maps $(a, \infty)$ onto itself and reverses the orientation. (1) is transformed into

$$
u^{(n)}+(-1)^{n}(\xi-a)^{-2 n} p\left(a+(\xi-a)^{-1}\right) u=0,
$$

and $(1)$ is $(k, n-k)$-disconjugate and disfocal on $(a, \infty)$ if and only if $(50)$ is $(n-k, k)$-disconjugate and disfocal on $(a, \infty)$. Let $c_{k}(\alpha)$ be the right hand side of (47), i.e., let

$$
(x-a)^{\alpha} \int_{x}^{\infty}(t-a)^{n-\alpha-1}|p(t)| d t \leqq c_{k}(\alpha), \quad a<x<\infty
$$

ensure the $(k, n-k)$-disfocality of (1). Similarly

$$
(\xi-a)^{\alpha} \int_{\xi}^{\infty}(t-a)^{n-\alpha-1}\left\{(t-a)^{-2 n}\left|p\left(a+(\xi+a)^{-1}\right)\right|\right\} d t \leqq c_{n-k}(\alpha)
$$

implies that $(50)$ is $(n-k, k)$-disfocal and disconjugate on $(a, \infty)$ hence $(1)$ is $(k, n-k)$-disfocal and disconjugate on $(a, \infty)$. By setting $t-a=(s-a)^{-1},(51)$ reduces to

$$
(\xi-a)^{\alpha} \int_{a}^{a+(\xi-a)^{-1}}(s-a)^{n+\alpha-1}|p(s)| d s \leqq c_{n-k}(\alpha), \quad a<\xi<\infty .
$$

If we substitute $u-a=(\xi-a)^{-1}$, (52) is equivalent to

$$
(u-a)^{-\alpha} \int_{a}^{u}(s-a)^{n+\alpha-1}|p(s)| d s \leqq c_{n-k}(\alpha), \quad a<u<\infty .
$$

COROLLARY. If

$$
(x-a)^{-\alpha} \int_{a}^{x}(s-a)^{n+\alpha-1}|p(s)| d s \leqq c_{n-k}(\alpha), \quad a<x<b
$$

then (1) is $(k, n-k)$-disfocal on $(a, b)$.

For $b=\infty$, the corollary was proved above. When $b$ is finite, we may define $\tilde{p}(x)=p(x)$ for $a \leqq x \leqq b$ and $\widetilde{p}(x) \equiv 0$ for $b<x<\infty$. Then (53) holds, with $\widetilde{p}$ replacing $p$, on $(a, \infty)$ and the corresponding equation is $(k, n-k)$-disfocal on $(a, \infty)$, in particular on $(a, b)$. 
Similarly, the necessary conditions (35) of Nehari are transformed to

$$
x^{-\alpha} \int_{a}^{x} s^{n+\alpha-1}|p(s)| d s \leqq(n-1)^{2}(k-1) !(n-k-1) ! / \alpha
$$

for $0<\alpha \leqq n-1$. For $n=2$ see [14, p. 432] and for $n=4, p<0$, see Theorem 3.2 of [6].

Finally we obtain conditions specific for a compact interval. The transformation $\xi=(x-a) /(b-x)$ maps $(a, b)$ onto $(0, \infty)$ and keeps the orientation. (1) is transformed into

$$
u^{(n)}+(b-a)^{n}(1+\xi)^{-2 n} p((b \xi+a) /(\xi+1)) u=0, \quad 0<\xi<\infty
$$

and (1) is $(k, n-k)$-disconjugate on $(a, b)$ if and only if (55) is $(k, n-k)$-disconjugate and disfocal on $(0, \infty)$. By $(47)$, if

$\left.\xi^{\alpha} \int_{\xi}^{\infty} t^{n-\alpha-1}\left\{(b-a)^{n}(1+\xi)^{-2 n}\right)|p((b t+a) /(t+1))|\right\} d t \leqq c_{k}(\alpha), \quad 0<\xi<\infty$

then $(55)$ is $(k, n-k)$-disconjugate and disfocal on $(0, \infty)$. Substituting $t=(s-a) /(b-\varepsilon)$ and $\xi=(x-a) /(b-x)$ we obtain the sufficient condition.

$$
\begin{gathered}
\left(\frac{x-a}{b-x}\right)^{\alpha} \int_{x}^{b}(s-a)^{n-\alpha-1}(b-s)^{n+\alpha-1}|p(s)| d s \leqq c_{k}(\alpha)(b-a)^{n-1}, \\
a<x<b,
\end{gathered}
$$

for $(k, n-k)$-disconjugacy. By using (53) instead of (47) for equation (55), we can exchange the roles of $a$ and $b$ in (56).

For $\alpha=0,(56)$ gives a weaker but simpler sufficient condition

$$
\int_{x}^{b}(s-a)^{n-1}(b-s)^{n-1}|p(s)| d s \leqq(b-a)^{n-1}(n-k-1) ! k !
$$

Levin found the same condition with the better constant $(b-a)^{n-1}(n-l v-1) !(k-1) !(n-1)[11]$.

Necessary conditions of similar type are available from (35).

\section{REFERENCES}

1. U. Elias, Oscillatory solutions and extremal points for a linear differential equation, Arch. Rat. Mech. Anal., to appear,

2. G. J. Etgen and C. D. Shih, Disconjugacy of third order differential equations with nonnegative coefficients, J. Math. Anal. Appl., 41 (1973), 420-425.

3. R. D. Gentry and C. C. Travis, Comparison of eigenvalues associated with linear differential equations of arbitrary order, Trans. Amer. Math. Soc., 223 (1976), 167-179. 4. R. Grimmer, Comparison theorems for third and fourth order linear equations, J. Differential Equations, 25 (1977), 1-10. 
5. R. Hadass, On the zeros of the solutions of the differential equation $y^{(n)}+p y=0$, Pacific J. Math., 31 (1969), 33-46.

6. H. Howard, Oscillation criteria for fourth order linear differential equations, Trans. Amer. Math. Soc., 96 (1960), 296-311.

7. I. T. Kiguradze, Oscillation properties of solutions of certain ordinary differential equations, Soviet Math. Dokl., 3 (1962), 649-652.

8. W. J. Kim, On the zeros of the solutions of $y^{(n)}+p y=0$, J. Math. Anal. Appl., 25 (1969), 189-208.

9. V. A. Kondratev, Oscillatory properties of solutions of the equation $y^{(n)}+p y=0$, Trudy. Moskov. Mat. Obsc., 10 (1961), 419-436.

10. A. C. Lazer, The behavior of solutions of differential equation $y^{\prime \prime \prime}+p y^{\prime}+q y=0$, Pacific J. Math., 19 (1966), 435-466.

11. A. Ju. Levin, A Fredholm equation with a smooth kernel and boundary value problems for a linear differential equation, Soviet Math. Dokl., 5 (1964), 1415-1419.

12. D. L. Lovelady, Oscillation and a class of odd order linear differential equations, Hiroshima Math. J., 5 (1975), 371-383.

13. Oscillation and even order linear differential equations, Rocky Mountain Math. J., 6 (1976), 299-304.

14. Z. Nehari, Oscillation criteria for second order linear differential equations, Trans. Amer. Math. Soc., 85 (1957), 428-445.

15. Non oscillation criteria for nth order linear differential equation, Duke J. Math., 32 (1965), 607-616.

16. Disconjugate linear differential operators, Trans. Amer. Math. Soc., 129 (1967), 500-516.

17. - Nonlinear techniques for linear oscillation problems, Trans. Amer. Math. Soc., 210 (1975), 387-406.

18. - Green's functions and disconjugacy, Arch. Rat. Mech. Anal., 62 (1976), $53-76$.

19. W. Simons, Some disconjugacy criteria for self adjoint linear differeniial equations, J. Math. Anal., 34 (1971), 445-463.

20. C. C. Travis, Comparison of eigenvalues for linear differential equations of order 2n, Trans. Amer. Math. Soc., 177 (1973), 363-374.

Received November 17, 1977 and in revised form June 17, 1978.

Carnegie-Melion University

Pittsburg, PA 15213

Current address: TeChNion Haifa, 32000 IsRael 



\title{
PACIFIC JOURNAL OF MATHEMATICS
}

\section{EDITORS}

\author{
DONALD BABBITT (Managing Editor) \\ University of California \\ Los Angeles, CA 90024 \\ HUGo ROSSI \\ University of Utah \\ Salt Lake City, UT 84112 \\ C. C. MOORE \\ University of California \\ Berkeley, CA 94720
}

J. DugundJI

Department of Mathematics

University of Southern California

Los Angeles, CA 90007

R. FinN and J. Milgram

Stanford University

Stanford, CA 94305

\section{ASSOCIATE EDITORS}
E. F. BECKENBACH
B. H. Neumann
F. WolF
K. YOSHIDA

\section{SUPPORTING INSTITUTIONS}

\author{
UNIVERSITY OF BRITISH COLUMBIA \\ CALIFORNIA INSTITUTE OF TECHNOLOGY \\ UNIVERSITY OF CALIFORNIA \\ MONTANA STATE UNIVERSITY \\ UNIVERSITY OF NEVADA, RENO \\ NEW MEXICO STATE UNIVERSITY \\ OREGON STATE UNIVERSITY \\ UNIVERSITY OF OREGON
}

UNIVERSITY OF SOUTHERN CALIFORNIA

STANFORD UNIVERSITY

UNIVERSITY OF HAWAII

UNIVERSITY OF TOKYO

UNIVERSITY OF UTAH

WASHINGTON STATE UNIVERSITY

UNIVERSITY OF WASHINGTON

The Supporting Institutions listed above contribute to the cost of publication of this Journal, but they are not owners or publishers and have no responsibility for its content or policies.

Mathematical papers intended for publication in the Pacific Journal of Mathematics should be in typed form or offset-reproduced, (not dittoed), double spaced with large margins. Please do not use built up fractions in the text of the manuscript. However, you may use them in the displayed equations. Underline Greek letters in red, German in green, and script in blue. The first paragraph or two must be capable of being used separately as a synopsis of the entire paper. Please propose a heading for the odd numbered pages of less than 35 characters. Manuscripts, in triplicate, may be sent to any one of the editors. Please classify according to the scheme of Math. Reviews, Index to Vol. 39. Supply name and address of author to whom proofs should be sent. All other communications should be addressed to the managing editor, or Elaine Barth, University of California, Los Angeles, California, 90024.

50 reprints to each author are provided free for each article, only if page charges have been substantially paid. Additional copies may be obtained at cost in multiples of 50 .

The Pacific Journal of Mathematics is issued monthly as of January 1966. Regular subscription rate: $\$ 72.00$ a year (6 Vols., 12 issues). Special rate: $\$ 36.00$ a year to individual members of supporting institutions.

Subscriptions, orders for numbers issued in the last three calendar years, and changes of address should be sent to Pacific Journal of Mathematics, P.O. Box 969, Carmel Valley, CA 93924, U.S.A. Older back numbers obtainable from Kraus Periodicals Co., Route 100, Millwood, NY 10546.

PUBLISHED BY PACIFIC JOURNAL OF MATHEMATICS, A NON-PROFIT CORPORATION

Printed at Kokusai Bunken Insatsusha (International Academic Printing Co., Ltd.). 8-8, 3-chome, Takadanobaba, Shinjuku-ku, Tokyo 160, Japan.

Copyright (C) 1979 by Pacific Journal of Mathematics Manufactured and first issued in Japan 


\section{Pacific Journal of Mathematics}

\section{Vol. 81, No. $2 \quad$ December, 1979}

Ersan Akyildiz, Vector fields and equivariant bundles ............... 283

Ehrhard Behrends, The centralizer of tensor products of Banach spaces ( $a$ function space representation) ......................... 291

Geoffrey R. Burton, Congruent sections of a convex body ............. 303

John Warnock Carlson, $H$-closed and countably compact extensions ...... 317

Robert Charles Carlson, Eigenfunction expansions for selfadjoint

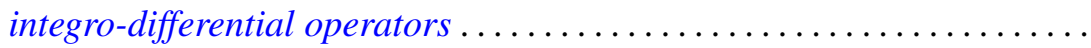

Robert Damiano, Coflat rings and modules.

Eric Karel van Douwen and Washek (Vaclav) Frantisek Pfeffer, Some properties of the Sorgenfrey line and related spaces ................

Uri Elias, Necessary conditions and sufficient conditions for disfocality and

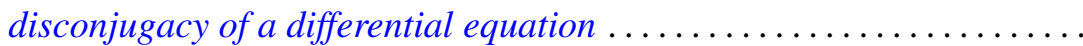

V. L. (Vagn Lundsgaard) Hansen, Polynomial covering spaces and homomorphisms into the braid groups .......................

Paul Hess, Dedekind's problem: monotone Boolean functions on the lattice

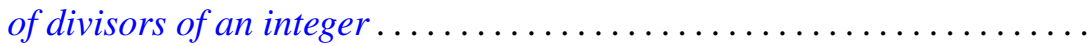

Alan Hopenwasser and David Royal Larson, The carrier space of a reflexive

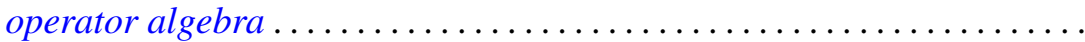

Kyung Bai Lee, Spaces in which compacta are uniformly regular $G_{\delta} \ldots \ldots$.

Claude Levesque, A class of fundamental units and some classes of

Jacobi-Perron algorithms in pure cubic fields...

Teck Cheong Lim, A constructive proof of the infinite version of the

Belluce-Kirk theorem ..........................

Dorothy Maharam and A. H. Stone, Borel boxes

Roger McCann, Asymptotically stable dynamical systems are linear

Peter A. McCoy, Approximation and harmonic continuation of axially symmetric potentials in $E^{3} \ldots \ldots \ldots \ldots \ldots \ldots \ldots$

Takahiko Nakazi, Extended weak-* Dirichlet algebras ....

Carl L. Prather, On the zeros of derivatives of balanced trigonometric polynomials ................................

Iain Raeburn, An implicit function theorem in Banach spaces...

Louis Jackson Ratliff, Jr., Two theorems on the prime divisors of zeros in completions of local domains...

Gloria Jean Tashjian, Cartesian-closed coreflective subcategories of

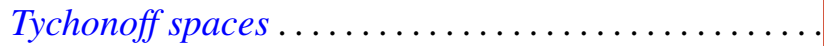

Stephen Edwin Wilson, Operators over regular maps.... . . 